\title{
FEA analysis and design optimization for a multifunctional piece of furniture
}

\author{
Georgeta Haraga ${ }^{1, *}$, and Adrian Mihai Goanță ${ }^{2}$ \\ 1 "Politehnica" University of Bucharest, Department of Engineering Graphics and Industrial Design, \\ Spl. Independenței No.313 Street, District 6, 060042, Bucharest, Romania \\ 2 "Dunărea de Jos" University of Galati, Engineering and Agronomy Faculty of Braila, Research \\ Centre for Mechanics of the Machines and Technological Equipments, Calea Calăraşilor Street, \\ 810017, Brăila, Romania
}

\begin{abstract}
The paper presents an original approach to the integration of computer aided design and finite element analysis for a multifunctional piece of furniture using CATIA Generative Structural Analysis workbench. Finite Element Analysis (FEA) application is an important engineering technique in the furniture industry. FEA is an accurate method for numerical solution of field problems. A major problem of mesh generation today is access to CAD geometry in a efficient and precise manner. Starting with a solid modelling for the creation of the desired piece of multifunctional furniture is used in this case, the automatic generation of the finite element meshes. Getting more accurate results for the mesh refinement process can be made by changing parameters. The article outlines the developed model for analysing the supporting structure of the armchair. This model provides simulating of the different loads and checking the raised stresses and deformation in the structures analysed. The main objective of this research is to evaluate strength of wooden cedar armchair for a person weighing $140 \mathrm{~kg}$ and for this the finite element analysis (FEA) has been used. Multifunctional furniture is the answer to the challenges contemporary lifestyle, creativity in design activities that require optimal space utilization and reducing costs.
\end{abstract}

\section{Introduction}

Furniture can be not only a functional product but also an artistic product and which can be associated with a form of decorative art. Furniture can contain different types of furniture pieces such as chairs, armchairs, tables, beds, cupboards and shelves. The pieces of furniture can be made using many materials like wood, metal and plastic. Thus, as an alternative to massive furniture, heavy and take up much space, the designers have brought into focus the multifunctional furniture, affordable, easy to install and operate.

Multifunctional furniture is considered a modern concept that can perform several functions simultaneously and which is based on recent knowledge of design, technology

* Corresponding author: g_haraga@yahoo.com 
and ergonomics. A set of furniture contains several kinds of individual pieces of furniture with multiple functionalities.

Many multifunctional furniture pieces are created from the need to satisfy the several requirements of the most demanding users. For these reasons, the conceptual model proposed for this study is a multifunctional armchair, which was created by necessity to have a working personal space. The armchair was designed in environmentally conscious manner, being made out of wood-based material. This study has been structured into two main parts: in the first part - Modelling a piece of furniture and in the second part Analysis of final model with FEA. The multifunctional piece of furniture proposed for the modelling and analysing is made in CATIA software, offering a realistic design that allows visualizing this model throughout its development. Produced of the company Dassault Systemes and distributed by IBM, CATIA offers many types modules with many applications in a variety of fields such as construction industry of machines, shipbuilding industry, automobile industry, aerospace, electrical engineering etc. [1]

\section{Modelling process}

Multifunctional furniture is known for extremely flexible design and that allows multiple uses. Furniture can be customized for the individual that needs to optimize space utilization.

The planning operation of space allocation is a specific optimization process, that involves allocating limited. The entire design process starts with the marketing research focused on several existing products and on potential user for the armchair [2]. Main criteria in furniture design are aesthetic, ergonomic and safety [3].

A significant number of benefits can be realized on during the development phase. The first part of this study involves designing of a multifunctional armchair with space optimization solutions for small room. Using CATIA Part module, the basic concept consists of two custom armrests of the chair with pockets of rectangular profile. The armchair is considered very durable being made from cedar wood, having all dimensions displayed in $\mathrm{mm}$ (W x H x D): $960 \times 875 \times 860$. A better image in terms of colour to this model can be obtained by applying the Properties command. Custom tables can be inserted into each pocket to provide space optimization solutions. The armrests of this piece of

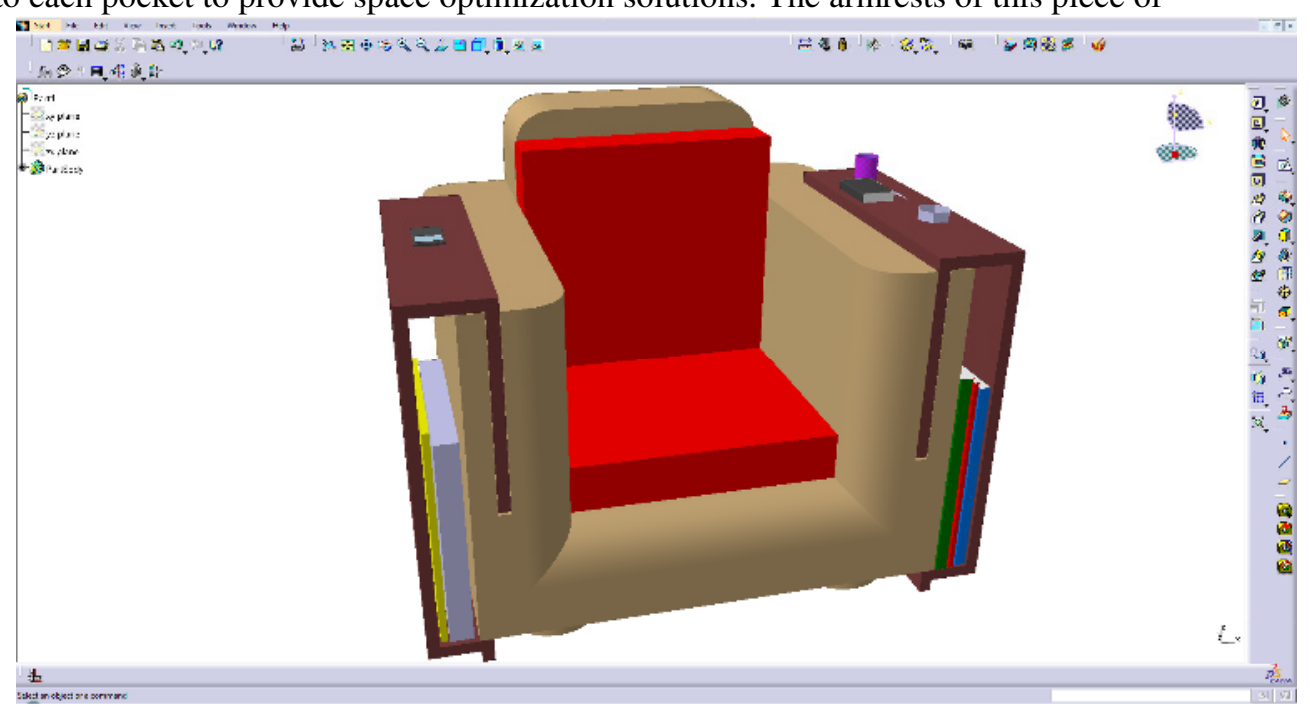

Fig. 1. Modeling of a multifunctional armchair. 
furniture can be equipped with wooden tables on each side as can be seen in Figure 1. Wooden tables can be used for rest periods, but it also, can be an excellent support for multiple everyday objects such as digital tablet or laptop, newspapers or magazines, books, coffee cups etc.

\section{Finite element analysis process}

Finite element analysis (FEA) is a significant computerized numerical method that provides approximate solutions for structural and thermal analysis in different applications areas.

FEA for a multifunctional piece of furniture (with/without custom wooden tables) can be done by using Generative Structural Analysis workbench of CATIA software.

A case of static analysis involves analysing the static boundary conditions of a CATIA Analysis document and consists of three main stages: Pre-processing, Processing and Postprocessing.

The Pre-processing step includes preparing to a specific part for Finite Element Analysis on the model and involves many stages such as: definition of type of analysis, definition of the material properties, generating mesh, applying restraints and loads.

The choice of material plays a crucial role in the design process and in this case cedar wood has been chosen. Cedar wood is often used for many applications in the furniture industry because it has a significant number of advantages, such as: lightweight, dimensionally stable, softwood and highly durable. Material properties are very important for any type of analysis and the mechanical properties of cedar wood such as: Young's modulus is $1.5 \mathrm{e}+010 \mathrm{~N} \_\mathrm{m}^{2}$ and Density is $750 \mathrm{~kg} \_\mathrm{m}^{3}$ can be seen in Figure 2 .

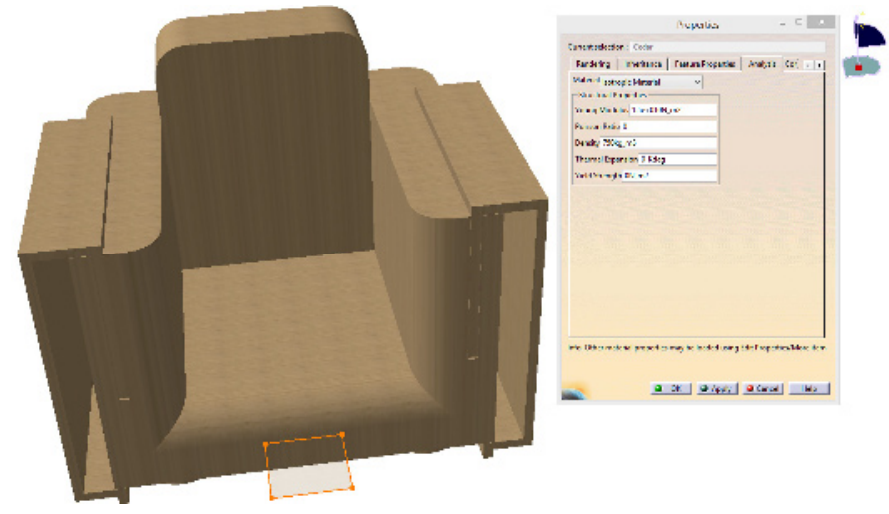

Fig. 2. The mechanical properties of cedar wood.

Generating Mesh represents meshing the part to create Finite Element model using elements and nodes. In both cases studied have been used "size" and "sag" and in the Octree Tetrahedral Mesh box have been entered the following values: Size $-10 \mathrm{~mm}$ and Sag - $2 \mathrm{~mm}$. "Size" refers to the elemental size of the mesh seen on the specific part and "sag" refers to the internal distance between the mesh size and the actual geometry of the part [4].

In the next step, there are established the restraints and loads on the model analysed. Any static analysis requires a linear static computation by referencing restraints and loads. The restraints can be achieved by the Restraints bar. According to the Clamp condition, the displacements are always zero in all three directions as shown in Figure 3.

The Processing is a second stage of the FEA process and includes the analysis for obtaining the correct solution (displacements, stresses etc.). This stage is an available 


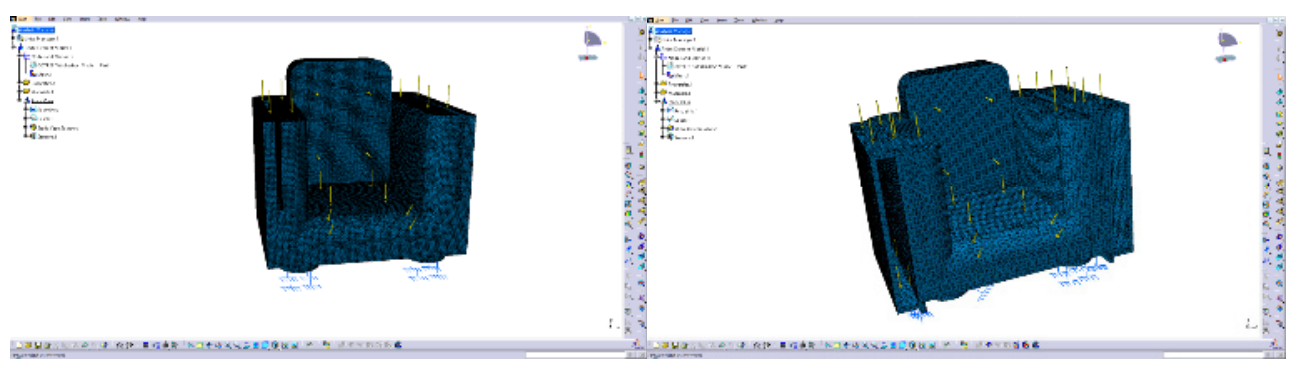

Fig. 3. Applying of the restraints and loads.

solution of the finite element problem provided by Pre-processing stage to find out the displacement values and to run the analysis and all these can be carried out by Compute command.

The Post-processing is the last stage of the FEA process and it refers graphical display of displacements and stresses for quick and easy interpretation, sorting and printing of results. The main toolbar in the Post-processing stage is called Image (Deformation, Von Mises Stress, Displacement). The Deformation option is used to view the deformed shapes according to the restraints and loads that were applied as can be seen in Figure 4.

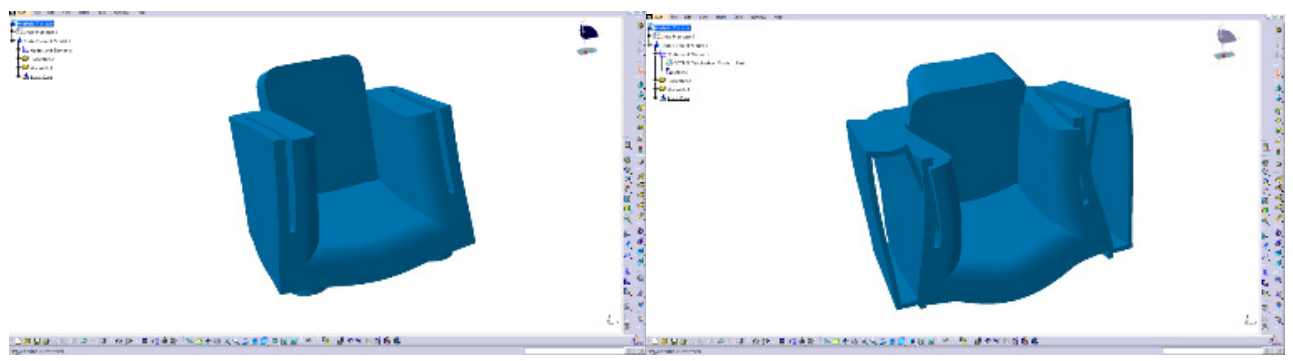

Fig. 4. Application of the Deformation command.

Stress analysis can be applied to entire structure or to a specific part $[5,6]$. The state of stress is described by the six Cauchy stresses (the normal stresses - $\sigma \mathrm{x}, \sigma \mathrm{y}, \sigma \mathrm{z}$ and the tangential stresses $-\tau \mathrm{x}, \tau \mathrm{y}, \tau \mathrm{z})$ which can vary from one point to another [7, 8]. Usually, the Cauchy stress tensor is used if the stress analysis of the model proposed is experiencing with small deformations when the differences in the distribution of stresses can be neglected.

The Von Mises stress is a combination of these and calculates values using the formula [9]:

$$
\sigma_{V M}=\sqrt{\frac{1}{2}\left[\left(\sigma_{x}-\sigma_{y}\right)^{2}+\left(\sigma_{x}-\sigma_{z}\right)^{2}+\left(\sigma_{y}-\sigma_{z}\right)^{2}+6\left(\tau_{x y}+\tau_{x z}+\tau_{y z}\right)^{2}\right]}
$$

As a general rule, the Von Mises Stress is only a positive number and in terms of principal stresses, $\sigma_{\mathrm{VM}}$ can also be written as [10]:

$$
\sigma_{V M}=\sqrt{\frac{1}{2}\left[\left(\sigma_{1}-\sigma_{2}\right)^{2}+\left(\sigma_{2}-\sigma_{3}\right)^{2}+\left(\sigma_{3}-\sigma_{1}\right)^{2}\right]}
$$

where $\sigma_{1}, \sigma_{2}, \sigma_{3}$ are namely principal stresses. 


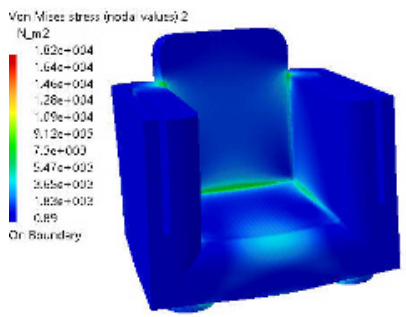

a. Mesh 1 (linear finite elements - Concept 1 )

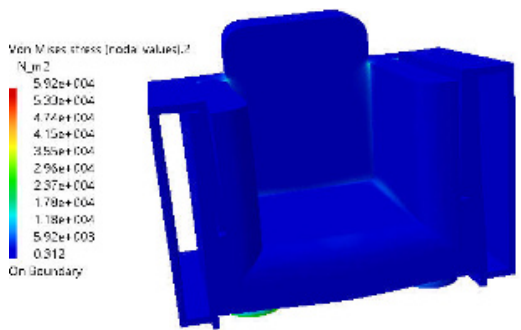

c. Mesh 3 (linear finite elements - Concept2)

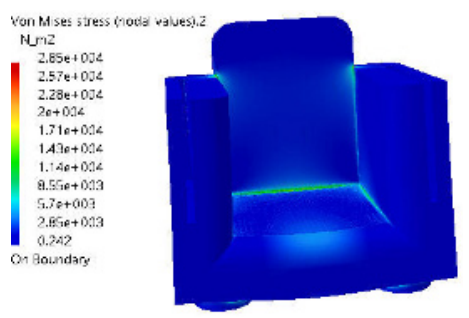

b. Mesh 2 (parabolic finite elements Concept1)

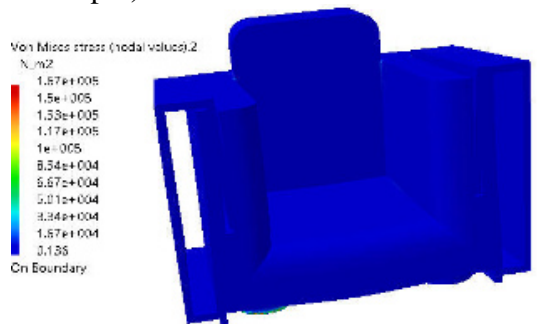

d. Mesh 4 (parabolic finite elements Concept2)

Fig.5. The von Mises stress (Static Case Solution 1 - Mesh 1, 2, 3, 4-a, b, c, d).

The Von Mises stress is the following icon from the Image toolbar. It is very important to understand that Von Mises stress is used by designers to check whether their design will withstand a given load condition [11].

The mesh refinement process can be made by changing global parameters can be seen in the Figure 5. In the first two cases analysed for Concept 1, Mesh 1 has the global mesh parameters ( size $=10, \mathrm{sag}=2$ ) with linear finite elements (145348 nodes, 651125 elements) and the global error rate of the analysis is $\varepsilon_{\mathrm{p} \text { initial }}=20.72 \%$, respectively, Mesh 2 has the global mesh parameters ( size $=10$, sag=2) with parabolic finite elements (1004303 nodes, 651125 elements) and the global error rate of the analysis is $\varepsilon_{\mathrm{p} \text { final }}=4.17 \%$. In the second two cases analysed for Concept 2, Mesh 3 has the global mesh parameters (size =10, sag=2) with linear finite elements (227259 nodes, 984167 elements) and the global error rate of the analysis is $\varepsilon_{\mathrm{p} \text { initial }}=27.25 \%$, respectively, Mesh 4 has the global mesh parameters ( $\operatorname{size}=10$, sag=2) with parabolic finite elements (1541050 nodes, 984167 elements) and the global error rate of the analysis is $\varepsilon_{\mathrm{p} \text { final }}=12.02 \%$.

The Displacement option allows viewing translational displacement of the model based on the loads that were applied upon it [12]. Displacements, Precision, Principal Stress distributions options are used along with a rich Colour Palette as shown in Figure 6.

\section{Conclusion}

The aim of the proposed in this paper is to design an armchair which has a study space optimization solution and make finite elements analysis for the supporting structure of an armchair with multifunctional facilities, taking into account the characteristics of its components. This paper is focused on the finite element analysis (FEA) of a multifunctional armchair having the following main objectives: choosing an the eco-friendly material that is quite frequently used in the furniture industry; adaptive mesh refinement; visualizing structural analysis results; reducing the global error rate, providing reasonable estimates. 


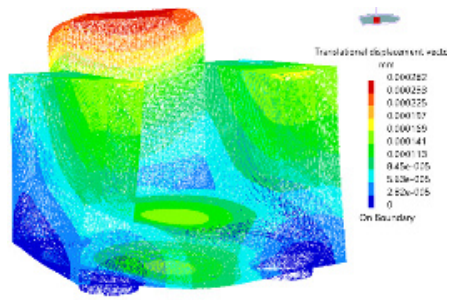

a. The Displacement option - Concept 1

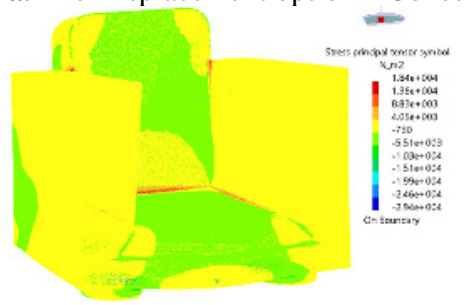

c. The Principal stress option - Concept 1

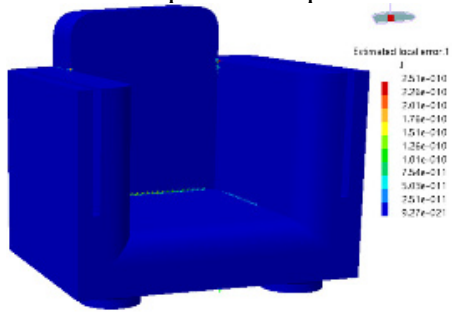

e. The Precision option - Concept 1

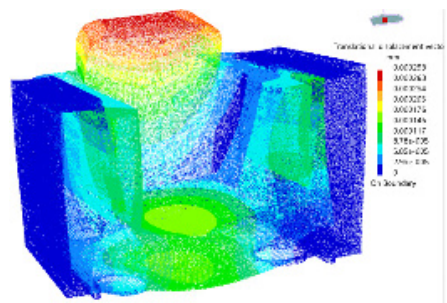

b. The Displacement option - Concept 2

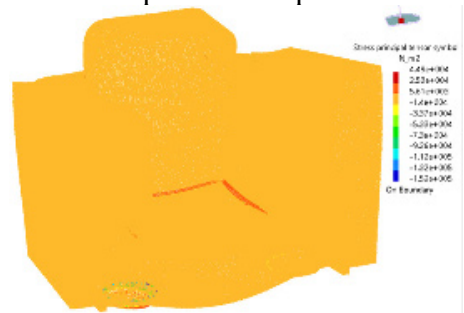

d. The Principal stress option - Concept 2

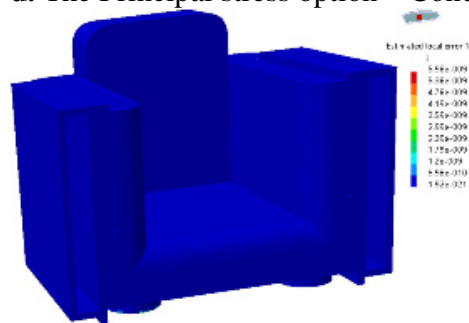

f. The Precision option - Concept 2

Fig.6. The Displacement, the Principal stress and the Precision options.

Making high-quality computations in the most automatic way possible was one of the main goals of the present paper. The final results of stress distribution and deformation are submitted for the analysis for this piece of multifunctional furniture. In FEA, there is an interpret the results from the color-coding. The red color shows the maximum deformation areas and the blue color shows the minimum deformation areas.

The mesh control is an important step in the Post-processing stage. The mesh parameters (geometric parameters - size and absolute sag and type of the finite element linear or parabolic) have a significant influence on the accuracy of numerical analysis.

The initial results for the global error rate of the analysis obtained in these case studies, are: Concept 1: $\varepsilon_{\text {plinitial }}=20.72 \%$ (Mesh 1); Concept 2: $\varepsilon_{\text {p2initial }}=27.25 \%$ (Mesh 3). For 3D models discretized with linear finite elements, the global error rate are in the range $27 \%$ $20 \%$, percent error that cannot be accepted. Under these circumstances, our goal is to reduce the percent $(\%)$ the global error rate of the analysis. Reducing of the global rate error can be done by changing the global parameteres and type of element. For Concept 1, the error has been reduced to $\varepsilon_{\text {p1final }}=4.17 \%$ (Mesh 2) that is substantially less than the $20.72 \%$ and for Concept 2, the error has been reduced to $\varepsilon_{\mathrm{p} 2 \text { final }}=12.02 \%$ (Mesh 4) that is a significant less than the $27.25 \%$.

Increasing the number of nodes can improve the accuracy of the results, but at the same time, it increases the solution time and cost. In recent years, have made a considerable progress in this direction, but there are still many problems remaining. 


\section{References}

1. A.M., Annals of Dunărea de Jos, Univ. Galați, XIV Mech. Eng., 1 9-12 (2013)

2. A. Fantu, L. Bârsan, Bulletin of Transilvania Univ. Brașov, România, 4 (53) 2 I 39-44 (2011)

3. I. Ntintakis, V. Iakovakis, G. Ntalos, J. Kechagias, Conference on Current Issues in Global Furniture 14-22 (2013)

4. G. Haraga, D. Ghelase, L. Daschievici, Computer Eng. and Appl. (CEA '16), ISI Proceding, Barcelona, Spain, 217-220 (2016)

5. CATIA V5 Fundamentals, EDU-CAT-E-V5R15 (2005)

6. D., Zahariea, Internat. J. of Modern Manufact. Techn. 3 No.2 117-122 (2011)

7. http://www.cadcamlab.org/courseware/PDFs/Stress\%20Analysis.pdf

8. https://www.capinc.com/2014/02/12/frequently-asked-questions-on-von-mises-stressexplained

9. http://www.sdcpublications.com/pdfsample/1-58503-227-1-2.pdf

10. http://www.learnengineering.org/2012/12/what-is-von-mises-stress.html

11. https://en.wikipedia.org/wiki/Cauchy_stress_tensor

12. http://www.cadcamlab.org/course/images/Example_Manuals_2006/Stress\%20Analysis \%20R14.pdf 